\title{
BAND THEORY OF MAGNETISM IN METALS IN CONTEXT OF EXACTLY SOLUBLE MODEL
}

\author{
DANIEL C. MATTIS \\ IBM Watson Research Center, Yorktown Heights, New York
}

(Received 13 October 1964)

\begin{abstract}
The role of spatial forces, and of Hund's rule (exchange) forces, and the validity of the Hartree-Fock approximation in the band theory of magnetism of metals is reviewed. Some reasonable conjectures which have been made elsewhere by the author and others are examined in the light of an exactly soluble model of interacting electrons in one dimension. It is shown that within the framework of validity of this model, spatial i.e., direct (Coulomb) forces do not influence the spin susceptibility, and conversely, Hund's rule exchange forces do not affect the dielectric constant or plasmon spectrum. It is recalled that the time-independent Hartree-Fock approximation yields an incorrect spectrum of elementary excitations, and it is also shown explicitly that the magnetic susceptibility calculated in this approximation is incorrect. A paradox is noted, concerning whether "correlations" can in fact correct the errors in the H-F approximation. Finally, it is shown that when there are only space forces, such spin density waves as might be introduced in to the $H-F$ ground state of the model are in fact spurious, because they are not representative of the correlations which exist in the true ground state.
\end{abstract}

\section{Introduction to the Band Theory of Magnetism}

IF the band theory of magnetism in metals remains today in a confused state, it is only partly due to a lack of solid quantitative results in many-body quantum mechanics. Another, and less justified, reason for the ambiguities and equivocation in the literature arises from the unfortunate fact that "exchange" is at best a relative concept, entirely dependent on the particular representation (or complete set of states) in which it is described. A final difficulty arises from the prevalent use of the Hartree-Fock (H-F) approximation in the solution of model Hamiltonians. In this approximation the ferromagnetic or other magnetic ordered states are overemphasized relative to the nonmagnetic states and, moreover, the excitation spectrum is incorrect.

Let us first discuss these problems qualitatively, starting with the last on e. The inadequacy of the $\mathrm{H}-\mathrm{F}$ approximation in the many-body problem is already well known in another context. Almost thirty years ago, Bardeen [1] showed that if the quasi-particle spectrum of a metal was calculated within the framework of H-F theory, the density of states at the Fermi surface, $\mathrm{N}(0)$, vanished - with disastrous consequences in the theory of the electronic specific heat and electrical conductivity of metals. Now that the structure of many-body perturbation theory is better understood, it is known that higher orders in the perturbation expansion (vaguely described by the generic name of "correlations") restore a finite value to $\mathrm{N}(0)$ even when they contribute little to the over-all energy [2].

As for "exchange", it should be clear on physical grounds that the sort of exchange responsible for the ordered spins in magnetic metals must be either weak or non-existent in the majority of the other, nonmagnetic, metals, and so can have little to do with the ubiquitous long-ranged Coulomb interaction. This 
could not yet have been clear to Bloch when, in the early days of the quantum theory of solids, he proposed the first band theory of ferromagnetism [3], basing it on just the spatial Coulomb repulsion equation (I . 4) between otherwise free electrons. But Pauli [4] commented: "Under those conditions (of low concentration, etc.), in fact, the approximation used by Bloch is rather bad; one must, however, consider as proved his more general result, which is, that ferromagnetism is possible under circumstances very different from those in which the Heitler-London theory is applicable; and that it is not sufficient, in general, to consider merely the signs of the (nearest-neighbor) exchange integrals."

A possible mechanism for magnetism soon became apparent from atomic structure calculations. It was found that the atomic, or "Hund's rule", magnetism was predicted at least qualitatively correctly by the Hartree-Fock approximation, al though the low-lying terms could not be described by single determinants but involved linear combinations of determinants. Qualitatively, they could easily be described in the vector model, a fact already well known to atomic spectroscopists on purely empirical grounds before the introduction of modern quantum mechanics. Configuration interactions (i.e., additional correlations) could be included to improve the accuracy of the variational ground and excited states; but the simplest and most primitive vector model of atomic structure should be sufficient for the theory of magnetism at the present time, considering the magnitude of the other uncertainties. Thus, Hund's rule exchange [5], otherwise known as atomic magnetism or the vector model is the force present in the various transition series metals which is absent in more common metals. In the latter, the unfilled shells presumably extend over distances of the order of the interatomic spacing, and the orbital degeneracies are lifted by crystal field and banding effects so that Hund's rule is partially or totally quenched. In the magnetic metals, on the other hand, the unfilled magnetically active shells are inner shells, and therefore have less nearestneighbor overlap. Thus, there is a strong, effectively spin-dependent, force equation (II.5) tending to magnetize these bands, a force which acts on two electrons when they are on the same atom and tends to align their spins, and which is of negligible magnitude when they are on separate atoms. This is the description of exchange in the representation of antisymmetrized products of Wannier functions, in which the occupation of each orbital of each atom or crystal cell can be specified in a given configuration.

Unfortunately, when the Coulomb interaction is expanded in a complete set of antisymmetrized products of Bloch functions, equations (II.8)-(II.11), some terms appear in the H-F approximation which have also been denoted "exchan ge forces", and which were precisely the terms Bloch had originally presumed responsible for ferromagnetism. This sort of "exchange" is present in all metals and therefore is not specific to ferromagnetics. If taken seriously, it predicts in addition to the vanishing density of states at the Fermi surface $N(0)=0$, an enhanced Pauli spin susceptibility which can in fact become infinite if $\mathrm{m}^{*}$, the band effective mass, is sufficiently large. In such a case the material is presumed spontaneously ferromagnetic, although it might also be unstable against some ordered antiferromagnetic configuration. We have already mentioned that the first result, concerning $\mathrm{N}(0)$, is incorrect. In fact the second result is incorrect also, as can be shown indirectly. In three dimensions one can analyze a class of interactions donated "separately symmetric" under the pairwise interchange of electronic $x^{-}, y-$, and $z$-coordinates, and solve for the total spin quantum number of the ground state. If one treated such potentials approximately, as one does the Coulomb force, he would find "exchange" contributions and an enhancement of the Pauli spin susceptibility. However, the exact result is that in the ground state the total spin vanishes just as for noninteracting particles [6]. Thus, correlations must go a long way towards cancelling the enhanced Paul spin susceptibility and will keep it finite, just as they go a long way toward restoring the unperturbed density of states at the Fermi surface. A similar point was the substance of Wigner's famous rebuttal of Bloch's theory [7], although his arguments then were, of necessity, largely in tuitive.

Today there is a resurgence of interest in the ferromagnetic metals, and in the antiferromagnetic ones, because of the peculiar problems they pose both to theory and to experiment. There has been discussion of the nature of spin waves in metals, and even of the elusive magnon-magnon interaction and of the thermodynamics above and near the critical temperature where the ordered state disappears. Yet, much of this work has been based on the idealizations of the Bloch model outlined in the previous paragraph, which we believe to be wrong. We believe that for a theory to be meaningful, it must deal with electrons in two or more degenerate bands and never in the one-band approximation. It must deal with the Coulomb repulsion (both the long-ranged part which yields the long wavelength plasma oscillations, and the short-ranged part 
which helps preserve electrical neutrality of each atomic cell) to much better accuracy than the H-F approximation. And finally, a meaningful theory must include intra-atomic intra-band matrix elements [e.g. (II.5)], such as would be capable of producing pure atomic magnetism if all metallic bonding were eliminated [8].

We shall illustrate these points by means of an exactly soluble model. In this model, the kinetic energy of the electrons is linearized (as in Dirac's relativistic theory), but the potential energy and interactions are left arbitrary. The linearization of the kinetic energy is sufficient to make the random phase approximation (otherwise known as time-dependent Hartree-Fock theory) an exact procedure in one dimension only, although this does not guarantee the validity of the H-F time-independent approximation. We shall see that the exact solution is in agreement with the picture of magnetism we have discussed here and elsewhere [8,9], whereas the H-F approximation gives results similar to those of the Bloch theory [3], which we shall show to be entirely wrong - qualitatively as well as quantitatively. Therefore, while the present model is not a realistic theory of magnetism in three dimensions, it does serve notice that the $\mathrm{H}-\mathrm{F}$ approximation, with or without spin density waves [10], is suspect. A physical explanation might be that particles in plane-wave (Bloch) states are on the average a distance $\approx \mathrm{L}$ apart and therefore "exchange" forces among them must be partly, or totally, cancelled by "correlation" forces. The latter are outside the scope of the H-F approximation, and therein lies the rub.

\section{Soluble Model of Interacting Electrons}

Recently, Luttinger [11] published a model of spinless and massless one-dimensional, interacting, fermions. He found a singularity at the Fermi surface, compatible wi th the results of many-body perturbation theory. Unfortunately, in calculating the energies and wave functions of his model Hamiltonian, Luttinger fell prey to a subtle paradox inherent in quantum field theory and therefore did not achieve a correct solution of the problem he had himself posed. The paradox is one which had been anticipated some years earlier by Schwinger [12], who had noted that equal time commutators of density operators of a fermion field, which vanish identically in first quantization, cannot vanish once the existence of a ground state such as the filled Dirac sea - is merely postulated. However, Schwinger's work was unknown to the present author at the time when, together with Lieb [13], he discovered this difficulty in the Luttinger model and proposed an exact solution to the model based on the correct commutation relations. The following model is a natural generalization of the earlier one, and is based on the observation that spin density operators do commute with spatial density operators, and that the desirable extension of the model to include magnetic forces is in fact possible.

In first quantization, our model consists of the following Hamiltonian:

$$
\begin{aligned}
\mathscr{H} & =-i \sum_{n=1}^{N} \frac{d}{d x_{n}}+i \sum_{m=1}^{M} \frac{d}{d y_{m}} \\
& +\frac{1}{2} \lambda \sum_{n, n^{\prime}} V\left(x_{n}-x_{n^{\prime}}\right)+\lambda \sum_{n, m} V\left(x_{n}-y_{m}\right)+\frac{1}{2} \lambda \sum_{m, m^{\prime}} V\left(y_{m}-y_{m^{\prime}}\right) \\
& -\sum_{n, m} J\left(x_{n}-y_{m}\right) S_{n}{ }^{z} S_{m}^{2}-\mu_{B} \sum\left[S_{n}^{2} H\left(x_{n}\right)+S_{m}{ }^{2} H\left(y_{m}\right)\right]
\end{aligned}
$$

There are basically two types of electrons: the $x$ 's, with "kinetic" energy $-i \frac{d}{d x}$, and the $y$ 's, with "kinetic" energy $+i \frac{d}{d y}$. It is this linearization of the (usually quadratic) kinetic energy operators which allows the model to be solved for arbitrary potentials $V$ and $J$. However, one unsatisfactory feature is that $\mathscr{H}$ as it stands has no ground state, just as in the case of the Dirac equation. Aside from the one-dimensionality, there is another unsatisfactory feature in the model, from the point of view of magnetism: for the Hund's rule interaction between two particles we must take the anisotropic form $S_{1}{ }^{2} S_{2}{ }^{2}$ rather than 
the rotationally symmetric form, $S_{1} \cdot S_{2}$, in order that the model remain soluble. This would lead to error principally in calculations of the ferromagnetic spin wave spectrum; but this spectrum is one feature which, anyhow, we shall find it beyond the powers of the present model to describe [see discussion following equation (I V.11)]. Otherwise, the interaction with $J(r)=J$ for $|r|\langle a$ and $J(r)=0$ for $|r|>a$, should give a fairly reasonable representation of the Hund's rule potential described in the introduction. The interaction of the electrons' spins with a variable external field $H(x)$ is given, as usual, in terms of the Bohr magneton $\mu_{B}$.

The lack of ground state in the spectrum of (II.1) is now remedied by filling the Dirac sea. It is necessary to rewrite the Hamiltonian in second quantization, using rules which have been previously given [13]. The following Hamiltonian is therefore our real starting point:

$$
\mathscr{H}=\mathscr{H}_{0}+\mathscr{H}_{1}+\mathscr{H}_{2}+\mathscr{H}_{3}
$$

where

$$
\begin{aligned}
& \mathscr{H}_{0}=\sum_{\substack{a 11 k \\
m= \pm \frac{1}{2}}}|k|\left(c_{k m}^{*} c_{k m}+b_{k m}^{*} b_{k m}\right) \\
& \mathscr{H}_{1}=\frac{1}{2} \frac{\lambda}{L} \sum_{m, m^{\prime}= \pm \frac{1}{2}} v(p)\left[\rho_{1 m}(-p)+\rho_{2 m}(-p)\right]\left[\rho_{1 m^{\prime}}(+p)+\rho_{2 m^{\prime}}(+p)\right] \\
& \mathscr{H}_{2}=\sum_{p}|p| J(p) \mathscr{S}_{1}(-p) \mathscr{S}_{2}(p) \\
& \mathscr{H}_{3}=-\mu_{B} \sum \sqrt{|p|} h(p)\left[\mathscr{S}_{1}(p)+\mathscr{S}_{2}(p)\right]
\end{aligned}
$$

with $v(p), J(p)$, and $h(p)$ being real functions proportional to the Fourier transforms of the space functions $V(x), J(x)$, and $H(x)$. The fermion operators $c$ and $c^{*}$ refer to holes in the filled sea of negative energy, and $b$ and $b^{*}$ to particles in states of positive kinetic energy. In the ground state of $\mathscr{H}_{\mathrm{o}}$ for $N_{\circ}$ particles, there are present $b$ particles of both types of spin from $+k_{F}$ to $-k_{F}$, with $k_{F}=\frac{1}{4} N_{\circ} 2 \pi / L$, and no holes in negative energy states. The particle-number operator is

$$
\mathscr{N}=\sum_{\substack{m= \pm \frac{1}{2} \\ \text { all k }}}\left(b_{k m}^{*} b_{k m}-c_{k m}^{*} c_{k m}\right)
$$

and of course commutes not only with the entire Hamiltonian, but also with all operators $\rho_{i m}( \pm p)$ and $\mathscr{S}_{i}( \pm p)$, with $i=1,2$. We have not defined these as yet, and now proceed to do so. In the following, always assume $p \geq 0$, unless otherwise specified.

$$
\rho_{1 m}(p)=-\sum_{k<-p} c_{k m}^{*} c_{k+p m}+\sum_{-p \leq k<0} b_{k+p m}^{*} c_{k m}^{*}+\sum_{k \geq 0} b_{k+p m}^{*} b_{k m}
$$

and for negative momenta, we take the Hermitean conjugate of the above:

$$
\rho_{1 m}(-p)=\rho_{1 m}^{*}(+p)
$$


Similarly,

$$
\rho_{2 m}(p)=\sum_{k<-p} b_{k+p m}^{*} b_{k m}+\sum_{-p<k<0} c_{k+p m} b_{k m}-\sum_{k>0} c_{k m}^{*} c_{k+p m}
$$

and

$$
\rho_{2 m}(-p)=\rho_{2 m}^{*}(+p)
$$

We observe the following relation:

$$
\mathscr{N}=+\sum_{m= \pm \frac{1}{2}}\left[\rho_{1 m}(0)+\rho_{2 m}(0)\right]
$$

The spin operators $\mathscr{S}_{i}( \pm p)$ are

$$
\mathscr{S}_{i}(p)=\left(\frac{\pi}{|p| L}\right)^{\frac{1}{2}}\left[\rho_{i,+\frac{1}{2}}(p)-\rho_{i,-\frac{1}{2}}(p)\right]
$$

for both positive and negative $p$, and $i=1,2$. The reason for such normalization will soon become apparent. We next calculate commutators, which vanish in first quantization but which here have the following values [13] :

$$
\begin{aligned}
{\left[\rho_{1 m}(-p), \rho_{1 m^{\prime}}\left(p^{\prime}\right)\right] } & =\left[\rho_{2 m}(p), \rho_{2 m^{\prime}}\left(-p^{\prime}\right)\right] \\
& =\frac{p L}{2 \pi} \delta_{m m^{\prime}} \delta_{p p^{\prime}}
\end{aligned}
$$

for $p$ negative or positive. Also,

$$
\left[\rho_{1 m}(p), \rho_{2 m^{\prime}}\left( \pm p^{\prime}\right)\right]=0 .
$$

Using these basic relations, we deduce:

$$
\begin{aligned}
{\left[\mathscr{S}_{1}(-p), \mathscr{S}_{1}\left(p^{\prime}\right)\right] } & =\left[\mathscr{S}_{2}(p), \mathscr{S}_{2}\left(-p^{\prime}\right)\right] \\
& =\delta_{p p^{\prime}} \quad(p>0)
\end{aligned}
$$

and all other commutators of $\mathscr{S}$ 's (amongst themselves) vanish. The last equation is precisely that obeyed by bosons, and therefore we shall name these (inaptly, but conveniently) magnon raising and lowering operators. Unlike magnons in a Heisenberg model, these are longitudinal magnons, defined as follows:

$$
\mathscr{S}^{+}(p)= \begin{cases}\mathscr{S}_{1}(p) & \text { for } p>0 \\ \mathscr{S}_{2}(p) & \text { for } p<0\end{cases}
$$

and

$$
\mathscr{S}^{-}(p)= \begin{cases}\mathscr{S}_{2}(p) & \text { for } p>0 \\ \mathscr{S}_{1}(p) & \text { for } p<0\end{cases}
$$


These operators have very important features, which permit the model to be soluble. They commute with the electron density operators $\rho_{i}(p)$,

$$
\rho_{i}(p) \equiv \rho_{i,+\frac{1}{2}}(p)+\rho_{i,-\frac{1}{2}}(p)
$$

and both sets of operators (II.17) and (II.18) have in common the property, that each is either a pure raising operator, or a pure lowering operator of the kinetic energy Hamiltonian $\mathscr{H}_{0}$.

\section{Factorization of the Normal Modes}

Following the procedure of the earlier work [13], we shall now decompose the Hamiltonian into three noninteracting parts: a quasi-particle part, a plasmon part, and a magnon part. We shall solve only for the spectrum of the last-named, although the procedure can be repeated in straightforward fashion to obtain the plasmon and quasi-particle spectra. Because these will not affect the magnetic properties of the model, they will not be studied explicitly here. However, the factorization takes all correlations into account.

Thus, we write

$$
\mathscr{H}=\mathscr{H}_{\mathrm{qp}}+\mathscr{H}_{\mathrm{p} 1}+\mathscr{H}_{\text {mag }}
$$

arranging that the quasi-particle, plasmon, and magnon Hamiltonians all commute; the following definitions will ensure that they do.

$$
\begin{aligned}
& \mathscr{H}_{\mathrm{qp}}=\mathscr{H}_{0}-T_{\mathrm{pl}}-T_{\mathrm{mag}} \\
& \mathscr{H}_{\mathrm{pl}}=T_{\mathrm{pl}}+\mathscr{H}_{1}
\end{aligned}
$$

and

$$
\mathscr{H}_{\text {mag }}=T_{\text {mag }}+\mathscr{H}_{2}+\mathscr{H}_{3}
$$

It is obvious that they all add up to yield the proper Hamiltonian. The new quantities are:

$$
T_{\mathrm{pl}}=\frac{\pi}{L} \sum_{p>0}\left[\rho_{1}(p) \rho_{1}(-p)+\rho_{2}(-p) \rho_{2}(p)\right]
$$

and

$$
T_{\mathrm{mag}}=\sum_{\mathrm{a} 11 p}|p| \mathscr{S}^{+}(p) \mathscr{S}^{-}(p)
$$

The following important relations can be verified:

$$
\begin{aligned}
& {\left[\mathscr{H}_{\mathrm{qp}}, \rho_{i}( \pm p)\right]=0} \\
& {\left[\mathscr{H}_{\mathrm{qp}}, \mathscr{S}^{ \pm}( \pm p)\right]=0} \\
& {\left[\mathscr{H}_{\mathrm{pl}}, \mathscr{S}^{ \pm}( \pm p)\right]=0} \\
& {\left[\mathscr{H}_{\mathrm{mag}}, \rho_{i}( \pm p)\right]=0}
\end{aligned}
$$
Consider the eigenstates of $\mathscr{H}_{\mathrm{qp}}$ in which no bosons of either species are present, that is, the states
$\Phi_{i}$ for which 


$$
\rho_{1}(-p) \Phi_{i}=\rho_{2}(p) \Phi_{i}=\mathscr{S}^{-}( \pm p) \Phi_{i}=0 .
$$

with corresponding energies $E_{i}$.

Let the energy in each plasmon mode be $e_{\mathrm{pl}}(p) N_{p}$, where $N_{p}=0,1, \ldots$, and the energy in each magnon mode be $e_{\text {mag }}(p) n_{p}$, where $n_{p}=$ eigenvalue of $\mathscr{S}^{+}(p) \mathscr{S}^{-}(p)=0,1, \ldots ;$ so that each eigenstate of $\mathscr{H}$ can be labelled by $\left(i,\left\{N_{p}\right\}\right.$, and $\left.\left\{n_{p}\right\}\right)$, and has corresponding energy

$$
E=E_{i}+\sum_{p} e_{\mathrm{pl}} N_{p}+w_{\mathrm{pl}}+\sum_{p} e_{\mathrm{mag}} n_{\mathrm{p}}+w_{\mathrm{mag}}
$$

with $w_{\mathrm{pl}}$ and $w_{\text {mag }}$ the respective boson ground state energies.

It is now important to prove the following

Theorem: the energies $E_{i}$ are independent of the coupling constants $v(p)$ and $J(p)$ (and could, for example, be evaluated using the unperturbed Hamiltonian $\mathscr{H}_{\mathrm{o}}$, and the definitions above). Moreover, the energies $e_{\mathrm{pl}}, w_{\mathrm{pl}}$ (and a fortiori, the occupation numbers $N_{\mathrm{p}}$ ) are independent of $J(p), h(p)$ and $E_{i}$ (and could, for example, be evaluated using the Hamiltonian $\mathscr{H}_{0}+\mathscr{H}_{1}$ with $\left.J(p)=h(p)=0\right)$. Finally, the energies and occupations $e_{\text {mag }}$ and $w_{\text {mag }}$ and $n_{p}$ are independent of $\mathrm{v}(\mathrm{p})$ and $E_{i}$ and therefore could be evaluated using the Hamiltonian $\mathscr{H}_{0}+\mathscr{H}_{2}+\mathscr{H}_{3}$, i.e., by setting $v(p)=0$.

Proof: The last proposition in the theorem is the important one in the present context and therefore we prove it first. We note from (III.4) that only the operators $\mathscr{S}^{ \pm}( \pm p)$ appear in $\mathscr{H}_{\text {mag }}$, and in (III.7) we observe that these operators commute with $\mathscr{H}_{\mathrm{qp}}$ and with $\mathscr{H}_{\mathrm{pl}}$. From this it follows that the magnon energy eigenvalues $\Sigma e_{\text {mag }}(p) n_{p}+w_{\text {mag }}$ are independent of whatever parameters appear in the two other Hamiltonians, QED. The other propositions can be proved using almost identical arguments.

There results the important

Corollary: The magnetic properties (e.g. the magnetic spin susceptibility) are independent of the spatial forces $V(x)$ or $v(p)$. Conversely, the electrical properties (e.g. the dielectric constant) are independent of the explicitly spin-dependent forces parametrized by $J(x)$ and $H(x)$.

We have not yet computed the Pauli spin susceptibility $\chi_{\text {mag }}$ although this is done in the next section. The above corollary proves that the magnetic properties, including $\chi_{\text {mag }}$ will not depend on the electronic charge interactions, but only on the Hund's rule coupling, as alleged in the introduction. The factorization of normal modes which has been achieved in this section thus has had the simplying feature that only $\mathscr{H}_{\text {mag }}$ need be considered in calculating the magnetic properties of the model.

\section{Pauli Spin Susceptibility}

We first diagonalize $T_{\text {mag }}+\mathscr{H}_{2}$ by means of a canonical transformation. This changes $\mathscr{H}_{3}$ to $\mathscr{H}_{3}^{\prime}$; the latter is then incorporated into the Hamiltonian, and the exact energy levels in the presence of the external field are calculated.

We recall:

$$
T_{\text {mag }}+\mathscr{H}_{2}=\sum_{\text {all } p}|p|\left\{\mathscr{S}^{+}(p) \mathscr{S}^{-}(p)-\frac{1}{2} J(p) \mathscr{S}^{+}(p) \mathscr{S}^{+}(-p)-\frac{1}{2} J(p) \mathscr{S}^{-}(-p) \mathscr{S}^{-}(p)\right\}
$$

and

$$
\mathscr{H}_{3}=-\mu_{B} \sum_{\text {all } p} \sqrt{|p|} h(p)\left\{\mathscr{S}^{+}(p)+\mathscr{S}^{-}(p)\right\}
$$

with $\mathscr{H}_{\text {mag }}=T_{\text {mag }}+\mathscr{H}_{2}+\mathscr{H}_{3}$, equation (III.4).

(IV.1) may be diagonalized by a simple Bogolubov transformation, 


$$
\mathscr{S}^{ \pm}(p) \rightarrow \mathscr{S}^{ \pm}(p) \cosh u(p)+\mathscr{S}^{\mp}(-p) \sinh u(p)
$$

This transformation preserves, of course, the commutators (II.15), (III.6), etc. The function $u(p)$ is determined by requiring that nondiagonal operators $\left[\right.$ e.g. $\left.\mathscr{S}^{+}(p) \mathscr{S}^{+}(-p)\right]$ be eliminated from $T_{\text {mag }}+\mathscr{H}_{2}$. This leads at once to the equation,

$$
\tanh 2 u(p)=J(p)
$$

and

$$
T_{\text {mag }}+\mathscr{H}_{2} \rightarrow \sum_{\text {all } p}|p| \operatorname{sech} 2 u(p) \mathscr{S}^{+}(p) \mathscr{S}^{-}(p)+w_{\text {mag }}
$$

with the zero point energy equal to,

$$
w_{\text {mag }}=-\frac{1}{2} \sum_{\text {all } p}|p|[1-\operatorname{sech} 2 u(p)]
$$

This solution is well-behaved provided $|J(p)|<1$; otherwise, the energy becomes complex, which indicates an instability. We shall shortly see that this instability is equally reflected in the spin susceptibility function.

Under the transformation (IV.3), the interaction Hamiltonian becomes

$$
\mathscr{H}_{3} \rightarrow-\mu_{B} \sum_{\text {a11 } p} \sqrt{|p|} h(p)\{\cosh u(p)+\sinh u(p)\}\left\{\mathscr{S}^{+}(p)+\mathscr{S}^{-}(p)\right\}
$$

When these terms are eliminated by a second canonical transformation,

$$
\mathscr{S}^{ \pm}(p) \rightarrow \mathscr{S}^{ \pm}(p)+f_{p}
$$

with scalar function $f_{p}$ appropriately chosen to cancel the linear terms, we obtain a final result:

$$
\mathscr{H}_{\text {mag }} \rightarrow \sum_{\text {all } p}|p| \operatorname{sech} 2 u(p) \mathscr{S}^{+}(p) \mathscr{S}^{-}(p)+w_{\text {mag }}-\frac{1}{2} \sum_{\text {all } p} \chi_{\text {mag }}(p) h^{2}(p)
$$

with the susceptibility given by

$$
\chi_{\mathrm{mag}}(p)=2 \mu_{B}^{2}[1-J(p)]^{-1}
$$

We note that $\chi_{\mathrm{mag}}$ is temperature independent (as it should be), and has a pole at $J(p)=1$. This is in excellent agreement with our previously published semiclassical criterion for instability of the ground state of a three dimensional metal against ferromagnetism $[8,9]$, a criterion which reduces to

$$
J(0) \geq 1 \quad \text { for ferromagnetism }
$$

in the present problem. Unfortunately, we cannot study the ferromagnetic state itself, in the present model. For once the in equality (IV.11) is satisfied, an indefinite number of particles in the Dirac sea have to be repopulated and the problem is no longer well defined. 


\section{The Hartree-Fock Approximation}

Having established the correct result in the present model, that the magnetic properties depend only on the explicit magnetic Hund's rule coupling and not at all on the spatial (i.e. Coulomb) forces, we now must go back to the initial nonmagnetic Hamiltonian $\mathscr{H}_{0}+\mathscr{H}_{1}$, equations (II.3) and (II.4), and calculate magnetic properties in the old fashioned time-independent Hartree-Fock approximation. Insofar as the magnetic susceptibility is affected by the spatial forces $v(p)$, to that extent must the H-F approximation be distrusted. We shall also discuss the manner in which correlations have to act in order to effect a complete cancellation of the spurious dependence of the magnetic susceptibility on space forces.

The H-F approximation is never supposed to be valid for very strong potentials, therefore it is consistent to assume very weak coupling, i.e. $\lambda<<1$, and there are therefore a negligible number of holes (c-particles) in the Dirac sea. Of course, there will be plenty of "holes"' near the Fermi surface, but these are described in terms of the presence or absence of $b$-particles. The standard approximation now consists of retaining all terms in $\mathscr{H}_{1}$ which are diagonal in the particle-occupation numbers. By hypothesis of small $\lambda v(p)$, the occupation numbers of c-particles may be neglected to good accuracy. The part of the space Hamiltonian which is diagonal in the occupation number representation of positive energy plane wave (i.e. Bloch function) states is:

$$
\mathscr{H}_{D}=\sum_{k, m}|k| n_{k m}+\frac{\lambda}{2 L} \sum_{\substack{m \\ p}} v(p)\left\{\left(\sum_{k=0}^{\infty}+\sum_{k=-\infty}^{-p}\right)\left[n_{k m}\left(1-n_{k+p m}\right)\right]+\sum_{k=-p}^{0}\left(1-n_{k+p m}\right\}\right\}_{(\mathrm{V}, 1)}
$$

with $n_{k m}=b_{k m}^{*} b_{k m}$

Using $\Sigma n_{k m}=$ total positive energy particle number $\doteq$ constant (to within the negligible negative-sea hole population), we can eliminate uninteresting nonmagnetic constant terms from (V.1) and be left with the following:

$$
\mathscr{H}_{D}=\sum_{k m}|k| n_{k m}-\frac{\lambda}{2 L} \sum_{\substack{m \\ p}} v(p)\left[\sum_{k=0}^{\infty} n_{k m} n_{k+p m}+\sum_{k=-\infty}^{-p} n_{k m} n_{k+p m}+\sum_{k=-p}^{+p} n_{k m}\right]+\text { const }
$$

We assume $n_{k m}=1$ for $|k|<k_{F m}$ and $n_{k m}=0$ for $|k|>k_{F m}$, replace sums by integrals in the usual way, and obtain:

$$
\begin{aligned}
E_{D}^{\lambda}= & \frac{L}{2 \pi}\left(k_{F,+\frac{1}{2}}^{2}+k_{F,-\frac{1}{2}}^{2}\right) \\
& -\frac{\lambda L}{2}\left(\frac{1}{2 \pi}\right)^{2} \int_{-\infty}^{\infty} d p v(p)\left\{\left(\int_{-\infty}^{-p} d k+\int_{0}^{\infty} d k\right) f(k, m) f(k+p, m)+\int_{-p}^{+p} d k f(k, m)\right\}
\end{aligned}
$$

with $f(k, m)= \begin{cases}1 & |k|<k_{F, m} \\ 0 & |k|>k_{F, m}\end{cases}$

If we define $K$ and $k_{F}$ by

$$
K=k_{F,+\frac{1}{2}}-k_{F,-\frac{1}{2}}, \quad k_{F}=\frac{1}{2}\left(k_{F,+\frac{1}{2}}+k_{F,-\frac{1}{2}}\right)
$$

and rewrite $E_{D}^{\lambda}$ as a function of $K$ and $k_{F}$, viz., $E_{D}^{\lambda}\left(K, k_{F}\right)$, then the susceptibility can be calculated by introducing a static magnetic field $(H=$ constant in space and time), the interaction Hamiltonian reducing to 


$$
\mathscr{H}_{3} \propto-H \cdot L \cdot K
$$

The spin susceptibility $\chi_{\text {mag }}^{\lambda}$ can now be expressed in terms of the spin susceptibility for the noninteracting system, $\chi_{\mathrm{mag}}^{0}$,

$$
\chi_{\operatorname{mag}}^{\lambda}=\chi_{\operatorname{mag}}^{0} \frac{a(0)}{a(\lambda)}
$$

where

$$
\left.a(\lambda) \equiv \frac{d^{2} E_{D}^{\lambda}\left(K, k_{F}\right)}{d^{2} K}\right|_{K=0}
$$

It is an easy matter to verify that $a(\lambda)$ depends linearly on $\lambda$, and thus that the spin susceptibility depends in a nonvacuous way on the spatial forces, contrary to the theorem proved previously. A naively straightforward perturbation-theoretic analysis also leads one to conclude that the correlation energy (i.e. the correction to $E_{D}$ due to second-and higher-order perturbation theory) is $0\left(\lambda^{2}\right)$, and therefore cannot possibly cancel the linear dependence on $\lambda$. This patently incorrect conclusion presents us with a new paradox, one which it would be of interest to examine in the future [14].

\section{Spin Density Waves}

It is a simple matter to verify that the inclusion of any number of spin density waves does not improve the susceptibility, regardless of any apparent improvement in the H-F ground state energy. A H-F state with spin density wave gives nonvanishing expectation value to such operators as

$$
b_{k \pm Q, m}^{*} b_{k, m^{\prime}}
$$

with $m^{\prime}$ chosen to be either $m$ or $-m$ for all $k$. With the first choice, the susceptibility at wavevector $p=Q$ and magnetic field in the $z$-direction, will (spuriously) depend on $\lambda$. With the second choice, a transverse magnetic field ( $x$ - or $y$-direction) will induce a response in the system which, once again, will be spuriously modified to first order in $\lambda$. It has been claimed by Overhauser [15] that the H-F ground state of the electron gas with Coulomb interactions contains one or more spin density waves; in the present model, it is easy to see that although there are charge density fluctuations (i.e. the plasmon vacuumfluctuations in the true ground state), there are no spin density fluctuations unless Hund's rule forces are explicitly introduced, and therefore correlations of the type (VI.1) are spurious when there are only space forces, regardless of H-F theory. Nonvanishing correlations exist only when the explicitly magnetic coupling $\mathscr{H}_{\text {ma g }}$ is reintroduced, as may be verified (as a simple exercise in the use of the present model) by the calculation of the spin-spin correlation function $\left\langle\left|S_{i}(r) S_{j}\left(r^{\prime}\right)\right|\right\rangle$ in the ground state.

\section{References}

1. J. BARDEEn, Phys. Rev. 50, 1098 (1936). Note: the H-F approximation to which reference is made is the time-independent $\mathrm{H}-\mathrm{F}$ approximation. This is not to be confused with the so-called "timedependent H-F approximation", or "random phase approximation", the validity of which is not under question at the present time.

2. J. BARDEEn, op. cit., E. P. WOHLfARTh, Phil. Mag. 41, 534 (1950); M. GELl-MANN, Phys. Rev. 106, 369 (1957).

3. F. BLOCH, Z. Phys. 57, 545 (1929).

4. W. PAULI, article in Le Magnetisme (Proc. 6th Solvay Conf.) p. 212, Gauthier-Villars; Paris (1932). 
5. "Of the terms given by equivalent electrons, those with greatest multiplicity lie deepest, and of these the lowest is that with greatest L." - from G. HERZBERG, Atomic Spectra and Atomic Structure, p. 135, Dover, New York (1944). The first application of this concept to solid state physics is by J. C. SLATER, Phys. Rev. 52, 198 (1937).

6. E. LIEB and D. MATTIS, Phys. Rev. 125, 164 (1962).

7. E. WIGNER, Trans. F araday Soc. 205, 678 (1938). Exchange and correlations were subsequently examined quan titatively (for 2 electrons) by J. C. SLATER, H. STATZ and G. F. KOSTER, Phys. Rev. 91, 1323 (1953).

8. These points are amplified in the author's forthcoming book, The Theory of Magnetism: An Introduction to the Study of Cooperative Phenomena, Chap. 7. Harper \& Row, New York (1965).

9. D. MATTIS, Phys. Rev. 132, 2521 (1963).

10. See section VI. The introduction of spin into the H-F wavefunction is also known (in atomic physics) as the "extended H-F approximation."

11. J. M. LUTTINGER, J. Math. Phys. 4, 1154 (1963).

12. J. SCHWINGER, Phys. Rev. Letters 3, 296 (1959).

13. D. MATTIS and E. LIEB, J. Math. Phys. (To be published).

14. D. PINES, chapter in Solid State Physics (Edited by F. SEITZ and D. TURNBULL), Vol. 1. Academic Press, New York (1955), shows that correlations generally reduce magnetic susceptibility below value predicted by H-F theory. It is not clear, however, where his calculation stands in relation to the paradox brought out in the present text. See R. ROCKMORE, Phys. Rev. 125, 1778 (1962) for a more modern approach.

15. A. W. OVERhaU SER, Phys. Rev. 128, 1437 (1962); Phys. Rev. Letters 4, 462 (1960); J. Phys. Chem. Solids 13, 71 (1960). 